\title{
Las áreas académicas más relevantes en las revistas especializadas del campo educativo: análisis comparativo de la escala de Scimago
}

\author{
The most relevant academic areas in journals of \\ education: a comparative analysis of Scimago's rank
}

Juan C. Forero Buitrago y Paula K. Triviño Gaviria*

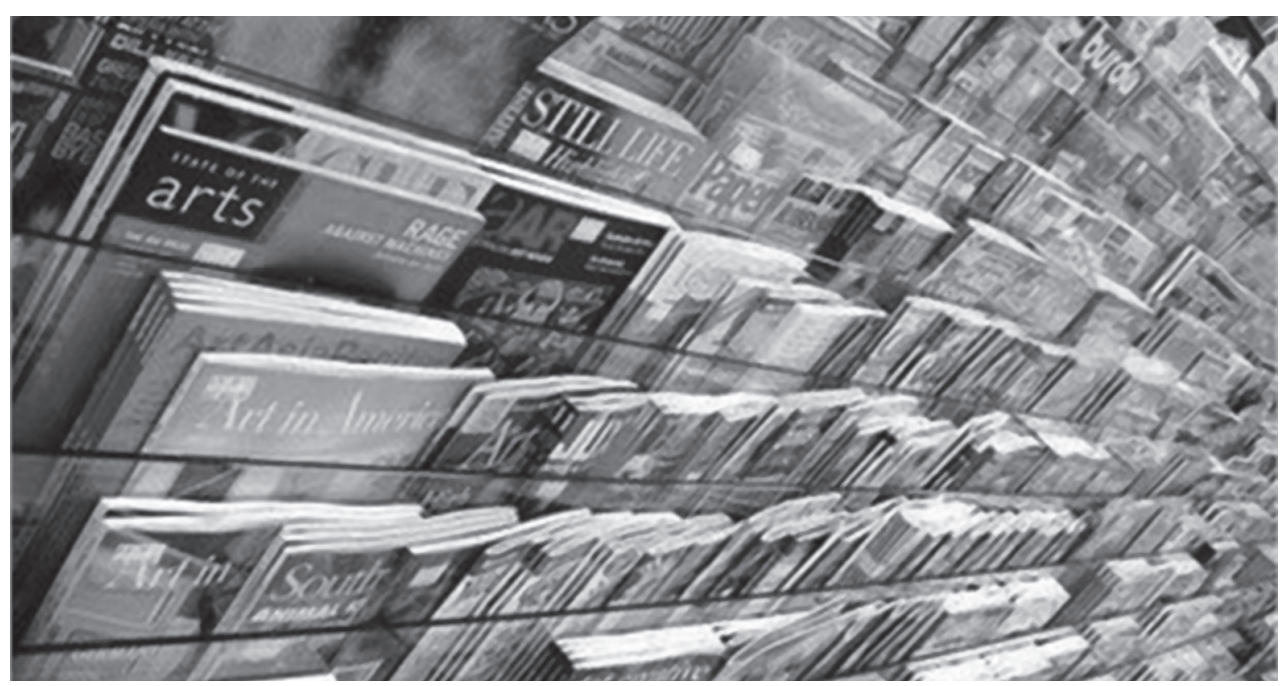

\section{Resumen}

Scimago es una de las instituciones encargadas de clasificar las revistas científicas más relevantes en la determinación del impacto académico en sus respectivos campos de estudio. Este trabajo busca realizar un análisis comparativo sobre las áreas académicas de las primeras 15 revistas especializadas clasificadas en Scimago para el año 2014 en educación. Este acercamiento comparativo se ubica en la necesidad de ahondar tanto en el conjunto de disciplinas dominantes sobre las cuales se inscriben estos

Citar este artículo como: Forero Buitrago, J. y Triviño Gaviria, P. (2016). Las áreas académicas más relevantes en las revistas especializadas del campo educativo: análisis comparativo de la escala de Scimago. Revista Papeles, 8(15), pp. 50-62.

Fecha de recibido: marzo 1 de 2016.

Fecha de aceptación: mayo 30 de 2016.

* $\quad$ Edición para publicación realizada por: Juan C. Forero Buitrago <jcforerob@unal.edu.co>; Paula K. Triviño Gaviria <pktrivinog@unal.edu.co>. Estudiantes de la Facultad de Economía de la Universidad Nacional de Colombia. Esta investigación fue el resultado de la cátedra Metodología de la investigación I, bajo la dirección del Prof. Enrique Ferrer-Corredor. Documento original por: Correos electrónicos: Diego H. Rodríguez Sosa $<$ dhrodriguezs@unal.edu.co >; Juan C. Forero Buitrago <jcforerob@unal.edu.co>; Laura A. Méndez Sarmiento $<$ laamendezsa@unal.edu.co>; Paola Solís Ruiz <psolisr@unal.edu.co>; Paula K. Triviño Gaviria < pktrivinog@ unal.edu.co>. 
documentos como en la pregunta: ¿Sobre qué se está investigando y publicando en educación?; haciendo indispensable ubicar, comparativamente, también tres revistas de nacionalidad colombiana en el mismo campo educativo. Para el cumplimiento de este propósito se seleccionaron unas variables determinadas (nacionalidad, idioma, área académica por revista y por índice, entre otras). Teniendo estas variables en cuenta se pueden hallar las relaciones y realizar las comparaciones entre los indicadores bibliométricos del campo especificado.

Palabras clave: revistas de educación, enseñanza de las ciencias, bibliometría, Scimago, educación.

\begin{abstract}
Scimago is the most relevant scientific journal ranking focused on determining the academic impact of these in their areas of study. This paper attempts to make a comparative analysis on the academic areas of the first 15 journals specialized on education, ranked by Scimago in the year 2014. This comparative approach states by the need to go into detail about the set of disciplines on which these documents were registered, and the question: what subjects are actually being investigated and published concerning the education? For this purpose, it is essential the analysis of three Colombian journals from the same education field. In order to achieve this goal, certain variables were selected (nationality, language, academic area determined by the journal and by its index, among others). Considering these variables, it could be found the existing connections and subsequently make comparisons between the bibliometric indicators within the specified category.
\end{abstract}

Key words: journals of education, science teaching, bibliometric, Scimago, education.

\title{
Criterios usados por Scimago Journal and Country Rank
}

En el siguiente apartado buscaremos dar a conocer los criterios por los cuales Scimago elige las revistas, ya que es importante resaltar qué parámetros utiliza para categorizarlas; permitiendo de este modo dar credibilidad y veracidad respecto a los ítems que enuncia en su página web. Las consideraciones generales tenidas en cuenta por Scimago para realizar su escala son las siguientes:

- Para el SIR (Scimago Institutions Rankings) es importante tener en cuenta tres indicadores como guía básica para aquellas revistas que integran el escalafón: de investigación, innovación y visibilidad en línea (online).

- Los indicadores de investigación, innovación y visibilidad de la web son medidos en rangos de 0 a 100, permitiendo así, hacer una evaluación comparativa entre las revistas, para poder posicionar cada institución.

- El mínimo de obras publicadas que se exige es de 100, las cuales deben estar contenidas en la base de datos SCOPUS ${ }^{1}$ durante el último año del período de tiempo.

- Lo que pretende Scimago como institución es precisar y encaminar hacia un solo punto la idea que se tiene de las instituciones por medio de un proceso manual exhaustivo para puntualizar el rango institucional.

1 SCOPUS $^{\odot}$ es propiedad de Elsevier Publishing Co., y es la base de datos de resúmenes e índices, de literatura con revisión por pares, más grande del mundo (Elseviers, s.f.). 
- La elección de las instituciones se realiza con resultados generados por un período de 5 años, incluyendo el año del cual se ejecuta la clasificación. Cuando se trata de indicadores web solo se toma el del período anual pasado.

- Se categorizan las instituciones por países para clarificar el origen de las revistas. Para este punto hay una excepción, ya que hay algunas instituciones, que son multinacionales, para las cuales no hay categorización en ningún país.

- Se busca homogeneizar las listas de las instituciones agrupadas por sectores institucionales.

- Cada indicador mencionado anteriormente tiene su propia fuente de información: para la innovación ha sido PATSTAT, para visibilidad de la web han sido Google y Ahrefs.

- Junto con los indicadores antes mencionados, el SIR tiene en cuenta las características científicas, económicas y sociales de las instituciones, organizándolas igualmente en una escala de 0 a 100. Esta caracterización no se ve influenciada por el tamaño de la institución (Scimago, 2014).

Con referencia al primer grupo de indicadores (los de investigación), que sirven de guía a las revistas que integran el escalafón SIR, enunciamos a continuación los criterios para su selección:

1. Un indicador dependiente del tamaño: es el número total de documentos publicados en revistas científicas indexadas en la base de datos de Scopus.

2. Un indicador de talla independiente: es la relación que tiene la institución de un país con instituciones extranjeras. Esta medición se hace por medio del análisis de lo que produce la institución y que está en vinculación con más de un país.

3. Un factor importante es el impacto normalizado de la producción dirigida, el cual ilustra una relación entre el impacto científico medio de una institución y de la media mundial a una puntuación de 1 , siendo 1 el punto medio. Por ejemplo, una institución punteada con un 0,7 será equivalente a $30 \%$ por debajo del promedio mundial; por el contrario, una puntuación del 1,4 será equivalente al $40 \%$ por encima del promedio mundial.

4. Para que las publicaciones sean clasificadas como de alta calidad o como aquellas que influyen más en el mundo, estas deben situarse en el primer cuartil (25\%) de las categorías clasificadas por SIRII.

5. Para clasificar las instituciones se utiliza el índice de especialización, el cual mide el nivel de concentración temática y la distribución de la producción científica de la institución, enumerándolas de 0 a 100 como en las demás categorías. Esta enumeración permite entender cuáles instituciones tienen tematizaciones más específicas y cuáles son más generales en los temas.

6. Para medir la cantidad de veces que un artículo ha sido citado se genera una tasa de excelencia presentada de forma porcentual.

7. Para medir la calidad y la cantidad de artículos de un autor perteneciente a una determinada institución se debe indicar el liderazgo científico.

8. Finalmente, se mide el número total de autores que aparecen en un volumen publicado por una institución durante un período determinado de tiempo (Scimago, 2014).

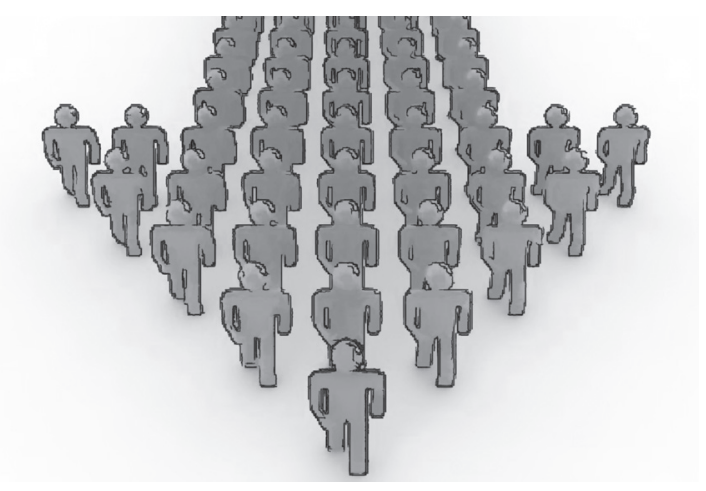




\section{Metodología}

Para el análisis de los campos académicos dominantes en las revistas especializadas en educación se escogió el último año en que Scimago ha realizado la clasificación: el 2014. Se tomaron las 15 primeras revistas especializadas de educación que se encuentran allí; además, se eligieron 3 revistas de educación de nacionalidad colombiana. Dentro de las 15 primeras se encontró el Handbook of the Economics of Education, siendo este un libro de series (book series) y no una revista especializada. Sin embargo, no se descarta, puesto que puede ser de gran conveniencia para el artículo contar con esa serie, ya que trata precisamente temas de economía y finanzas.

Dentro de las revistas colombianas de educación se tomaron las tres siguientes: la revista Magis de la Pontificia Universidad Javeriana, la cual hace parte del escalafón presentado por Scimago y que ocupa el puesto $804^{2}$; la revista Pedagogía y Saberes de la Universidad Pedagógica Nacional, ya que se encuentra nombrada por la escuela Normal de Colombia; y se toma como referencia la revista Papeles de la Universidad Antonio Nariño. Estas dos últimas revistas no se encuentran en el escalafón presentado por Scimago.

Para el análisis se realizó una tematización donde se tomaron como criterios volumen, número, último período de publicación de los artículos durante el 2014, nacionalidad, institución que la publica, temática o área de investigación según Scimago, Director in Chief, tabla de contenido, revisión por pares (peer review), tipo de acceso, formato y año de fundación. Para la tabla de contenido se escogieron los artículos publicados o entregas realizadas durante el período del 2014 correspondientes de junio a diciembre, o en su defecto, la última entrega.

\section{Tabla y análisis del número de artículos publicados en las revistas especializadas durante el año 2014}

Una de las variables que se consideró importante indagar acerca de las revistas especializadas fue el número de entregas y artículos publicados durante el año 2014, como se presenta en la tabla 1.

De acuerdo con la información presentada en la tabla anterior, y al analizar el número de artículos publicados y número de entregas realizadas durante el periodo de estudio, se concluyó que no se trata de la cantidad de artículos que están siendo producidos y

2 Se excluyeron la revista Academia de la Universidad de los Andes y el Journal of Science Education incluidas en la clasificación de Scimago. Por un lado, Academia es un caso excepcional, los artículos analizados hablan sobre precios, ampliación de negocios, belleza y moda. Por esto, no entendemos la razón por la cual se encuentra clasificada en la sub categoría de Edu- publicados por las distintas organizaciones a cargo de la revista, por el contrario, es cuestión de qué se está investigando y la calidad de dichas investigaciones. Partiendo de lo anterior, es aquí donde surge la cuestión de conocer si en verdad en Colombia se están haciendo investigaciones de calidad o solo se está hablando de un problema o temática. ¿Realmente Colombia se interesa por realizar investigaciones de calidad, innovar y proponer en los diferentes campos relacionados con

cación. Nos inclinamos a pensar, que puede ser por la comunidad a la que esta apunta: "La publicación está dirigida a estudiantes de posgrado, profesores y también estudiantes de pregrado." (Universidad de los Andes; septiembre 3 de 2014). Por otro, Journal of Science Education presenta una incongruencia de información que puede afectar el informe. 
Tabla 1. Lista de revistas seleccionadas: número de artículos y entregas por año

\begin{tabular}{lcc}
\hline \multicolumn{1}{c}{ Journal } & Artículos publicados por año & Entregas por año \\
\hline Journal of Research in Science Teaching & 52 & 10 \\
\hline Review of Educational Research & 18 & 4 \\
\hline Science Education & 45 & 6 \\
\hline Handbook of the Economics of Education & 0 & 0 \\
\hline Studies in Science Education & 7 & 2 \\
\hline Child Developmet & 177 & 6 \\
\hline American Educational Research Journal & 39 & 6 \\
\hline Journal of Educational Psychology & 96 & 4 \\
\hline Learning and Instruction & 63 & 6 \\
\hline Educational Researcher & 44 & 9 \\
\hline International Journal of Computer-Supported & 21 & 4 \\
Collaborative Learning & & 5 \\
\hline User modelling and User adapted interaction & 13 & 4 \\
\hline Journal of the learning Science & 29 & 4 \\
\hline Reading Research Quarterly & 24 & 10 \\
\hline Computers and Education & 233 & 2 \\
\hline Papeles & 16 & 2 \\
\hline Magis & 21 & 2 \\
\hline Pedagogía y Saberes & 23 & \\
\hline
\end{tabular}

* El Handbook of the Economics of Education hizo su última publicación en el año 2011 pero Scimago la tiene en cuenta en el ranking del año 2014. Para el año 2014 se obtiene 0 artículos, pero para el análisis de artículos, se toman los entregados en 2011.

la educación? Y ¿Qué están investigando las revistas de educación colombianas con respecto a las 15 primeras revistas especializadas clasificadas en Scimago?

\section{Idiomas y nacionalidad}

Es importante revisar detalladamente el entorno de los investigadores, a saber: nacionalidad, idioma de publicación, idioma de referencias bibliográficas, entre otros aspectos referenciados en este trabajo. La importancia de estos aspectos recae en caracterizar los factores sociales, económicos y culturales que determinan el éxito de las quince primeras revistas de educación en la clasificación, para así mismo realizar una comparación con las principales publicaciones científicas sobre educación de Colombia.

Los primeros quince puestos del escalafón están distribuidos en tres países: Estados Unidos, Reino Unido y Países Bajos. Estados
Unidos cuenta con el 47\% de participación en los quince primeros lugares, seguido por Reino Unido con un 33\% y Países Bajos con 20\%.

Gráfica 1. Participación de los países en la seleccionada

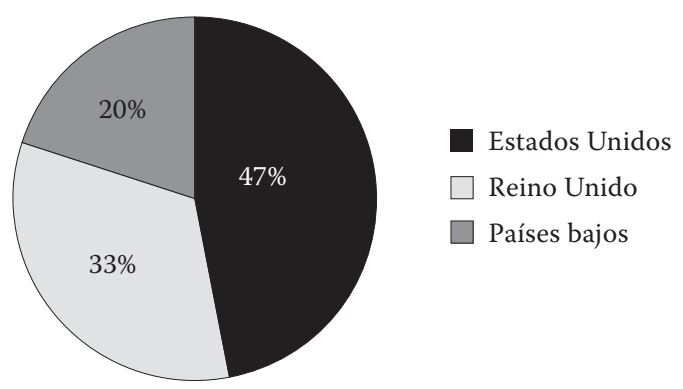

Fuente: elaboración propia 
Es necesario verificar estos datos de participación en la clasificación total que incluye 914 revistas. Estados Unidos cuenta con una participación del 30\% (305), Reino Unido con 36\% (330) y Países Bajos con 6.5\% (60); Colombia cuenta con el $0.33 \%$, con tres revistas.

Como es bien sabido, el inglés es el idioma predominante en temas académicos, y es de esperarse que las quince primeras revistas en el escalafón sean publicadas en inglés. Independiente de la nacionalidad de la revista, la mayoría (por no decir todas) son publicaciones en inglés. Esto no quiere decir que los artículos hayan sido originalmente escritos en inglés; ya que en una revista con escritores no-angloparlantes hay procesos de traducción posterior a la revisión por pares ${ }^{3}$.

El idioma tiene gran relevancia en el éxito de una revista; ya que el escalafón está realizado con algoritmos informáticos que clasifican las citaciones y el prestigio está determinado por el número de estas. Un artículo de buena calidad y de trabajo riguroso servirá de referencia bibliográfica para los artículos por venir, así que hay una directa correspondencia entre publicar en inglés y citar en inglés.

Otros países, como Alemania, Francia y Bélgica, aun teniendo en cuenta sus características como potencias mundiales, no aparecen en la clasificación sino hasta el puesto 43; cuando por encima están 6 revistas holandesas. No es extraño imaginar que el idioma realmente juega un papel importante en la producción de textos científicos relevantes para la academia, y que es la forma en que los países emergentes, como Colombia, deberían acercarse a la calidad y relevancia académica.

\section{Áreas de investigación y publicación}

Una de las más importantes variables que consideramos indagar acerca de las revistas de Scimago es el área académica sobre la cual esta trata. Si bien al realizar la investigación filtramos por la categoría educación, no hay un área del conocimiento específico que deseemos mirar, sino, por el contrario, observar cuáles son los temas y áreas sobre los que estas revistas están trabajando. Así, la primera división porcentual que hacemos dentro de las 15 primeras revistas es por área de conocimiento, según está indicado en Scimago. Sin embargo, dado que hay revistas que tratan a la vez diferentes disciplinas, la distribución se hará otorgando 10 puntos por área individual en el que se encuentre concentrando el tema global de la revista; y en caso de compartir esta ocupación con otras disciplinas, a cada una se les dará 5 puntos. Al final podremos ver la relación comparada de

3 Es importante anotar que, según nuestra investigación, las revistas colombianas clasificadas en el escalafón tienen varios o todos sus artículos escritos en inglés. las áreas generales, de las cuales se encargan las 15 primeras revistas en la escala de Scimago en la categoría de educación.

Tabla 2. Área académica según Scimago.

\begin{tabular}{lcr}
\hline \multicolumn{1}{c}{ Área mencionada } & Puntos obtenidos & \multicolumn{1}{c}{$\%$} \\
\hline Ciencias Sociales & 100 & $63 \%$ \\
\hline Artes y Humanidades & 5 & $3 \%$ \\
\hline Economía y Finanzas & 5 & $3 \%$ \\
\hline Psicología & 30 & $19 \%$ \\
\hline Medicina & 5 & $3 \%$ \\
\hline Ciencias de la computación & 15 & $9 \%$ \\
\hline
\end{tabular}

Fuente: elaboración propia

Gráfica 2. Distribución porcentual de la concentración temática según Scimago

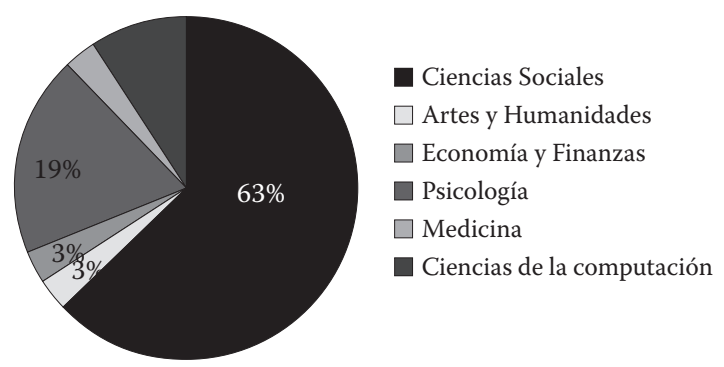

Fuente: elaboración propia 
Sin embargo, la tematización hecha por el grupo indica que hay una amplitud de áreas a las cuales se dedica la revista; dado, por ejemplo, que la categoría Ciencias Sociales o Social Sciences en Scimago parece recoger una gran cantidad de sub-disciplinas, tanto ideológicas, teóricas, como metodológicas, que para nosotros es importante simplificar y fragmentar de manera más profunda. Así, de acuerdo a nuestra categorización, hecha con base en las descripciones de las propias revistas, proponemos la siguiente división, teniendo en cuenta la misma distribución de puntos:

De esta manera se puede evidenciar que las áreas o disciplinas de preferencia investigativa casi se duplican de una categorización a otra

Tabla 3. Área académica específica según el grupo de investigación

\begin{tabular}{lcc}
\hline \multicolumn{1}{c}{ Área investigada } & Puntos & Porcentaje \\
\hline Ciencias Sociales & 10 & $7 \%$ \\
\hline Artes y Humanidades & 0 & 0 \\
\hline Economía y Finanzas & 5 & $4 \%$ \\
\hline Psicología & 5 & $4 \%$ \\
\hline Medicina & 0 & 0 \\
\hline Ciencias de la computación & 25 & $19 \%$ \\
\hline Enseñanza de la Ciencia & 15 & $11 \%$ \\
\hline Política educativa & 10 & $7 \%$ \\
\hline Literatura sobre educación & 20 & $15 \%$ \\
\hline Multi e Interdisciplinar & 35 & $26 \%$ \\
\hline Desarrollo infantil & 10 & $7 \%$ \\
\hline
\end{tabular}

Fuente: elaboración propia

Gráfica 3. Distribución porcentual por concentración temática específica según el grupo de investigación

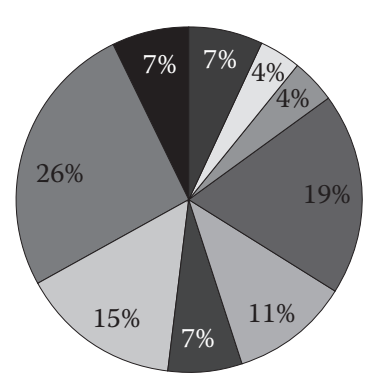

Fuente: elaboración propia
Ciencias Sociales $\square$ Artes y Humanidades $\square$ Economía y Finanzas $\square$ Psicología $\square$ Medicina

$\square$ Ciencias de la computación $\square$ Enseñanza de la Ciencia $\square$ Política educativa $\square$ Literatura sobre educación $\square$ Multi e interdisciplinar Desarrollo infantil (de Scimago a la nuestra), dando a entender, en primer lugar, que la categoría Social Sciences, en la cual se ubican más de la mitad de las revistas especializadas según Scimago, puede profundizar en otras áreas más específicas; por ejemplo, sobre los procesos y métodos de enseñanza de la ciencia, que en nuestra categorización ocupa un $16 \%$; la política educativa (11\%) y aquellas que se definen como multi e interdisciplinar (26\%). Así, específicamente referente al área de ciencias sociales como un conjunto de disciplinas, entre las que cabría rescatar, la historia, la sociología y la filosofía, solamente podemos clasificar el 7\%. Con la segunda caracterización hecha podemos ver las otras áreas en profundidad que componen esa gran categoría de Social Sciences usada por Scimago.

Por otro lado, las disciplinas de artes y humanidades y medicina, obtenidas en la primera categorización, desaparecen en la realizada por el grupo; en cambio, obtenemos que estas áreas están representadas por las especialidades de la literatura sobre educación y el desarrollo infantil específicamente.

Ahora bien, de acuerdo a ambas clasificaciones por área se puede decir que los temas investigativos predominantes en la clasificación más alta de Scimago corresponden al área de las ciencias sociales, con especialidades en los métodos de la enseñanza de la ciencia, procesos multi e interdisciplinares y política educativa. Lo que nos refiere que, en temas educativos, las ciencias sociales (que comprenden un amplio conjunto de disciplinas, técnicas y procesos) son el área por excelencia de investigación en educación. Sin embargo, aparece una disciplina relativamente joven en los últimos 5 puestos de nuestra selección de 15 revistas, que evidencia la rápida importancia que está asumiendo en los temas relevantes de investigación mundial: las ciencias de la computación y las tecnologías de la comunicación. La información y la enseñanza obtienen un puesto privilegiado al ser disciplinas con un amplio interés mundial y ocupando en nuestra 
clasificación una importancia del 19\%; siendo así el segundo tema o área académica más relevante en los temas generales de investigación en educación que trabajan las revistas más importantes a nivel mundial según Scimago.

Estas variables que estamos estudiando nos ayudan a formar un panorama frente a la pregunta que nos atañe en nuestro contexto inmediato: ¿Qué tanto las revistas colombianas de educación se acercan a los temas de preferencia investigativa con algunos de los índices bibliométricos más altos? Sin embargo, si tomamos las revistas con su categoría general no nos aportarán ningún dato del cual podamos analizar la cercanía entre lo que publica Colombia y lo que publica la cima de las más importantes revistas en educación, dado precisamente lo que hemos identificado como unas categorías demasiado amplias. Por ello mismo, a continuación, proponemos una clasificación temática por artículos publicados en los últimos volúmenes del año 2014 de cada revista. Permitiendo así, mirar con mayor especificidad qué se está publicando.

El siguiente es un cuadro de frecuencia donde se crearon categorías específicas para los temas que fueron encontrados en los artículos, así como para los niveles educativos mencionados por los autores. Se realizó a partir del título, resumen y las palabras clave.

Tabla 4. Tematización por artículos:

primeras 15 revistas en educación según la escala de Scimago.

\begin{tabular}{|c|c|c|}
\hline Categoría: Tema investigativo & Frecuencia & Descripción \\
\hline Sociedad y contextos & 34 & $\begin{array}{l}\text { Climas emocionales, la violencia y el conflicto, las relaciones de } \\
\text { origen y raza, las influencias familiares y las relaciones con los } \\
\text { padres. }\end{array}$ \\
\hline Tecnología y educación & 16 & $\begin{array}{l}\text { Las formas como diferentes plataformas tecnológicas, la Internet, } \\
\text { las redes sociales y otras tecnologías influyen en las maneras de } \\
\text { enseñar, aprender y desenvolverse en la escuela y el aula. }\end{array}$ \\
\hline $\begin{array}{l}\text { Métodos y metodología de } \\
\text { enseñanza y aprendizaje }\end{array}$ & 11 & $\begin{array}{l}\text { Debates de las formas cómo se enseña, del manejo de diferentes } \\
\text { tipos de información. }\end{array}$ \\
\hline $\begin{array}{l}\text { Razonamiento y pensamiento } \\
\text { matemático }\end{array}$ & 11 & $\begin{array}{l}\text { Artículos especializados en el pensamiento y lógica matemática, } \\
\text { sus aplicaciones y pedagogía. }\end{array}$ \\
\hline Desempeño académico & 9 & $\begin{array}{l}\text { Formas y análisis sobre las evaluaciones y auto evaluaciones, } \\
\text { factores que afectan el rendimiento académico. }\end{array}$ \\
\hline Desarrollo del aprendizaje & 9 & $\begin{array}{l}\text { Control del aprendizaje; experiencias y acompañamiento a los } \\
\text { estudiantes; educación formal e informal. }\end{array}$ \\
\hline Lenguaje y comunicación & 8 & $\begin{array}{l}\text { Análisis y artículos sobre la importancia del lenguaje, la comunica- } \\
\text { ción y sus medios; efectos de la lectura y la escritura. }\end{array}$ \\
\hline Análisis de los comportamientos & 8 & $\begin{array}{l}\text { Artículos sobre los comportamientos y prácticas de los estudian- } \\
\text { tes, profesores dentro del aula y la forma en cómo los niños actúan } \\
\text { frente a determinadas situaciones. }\end{array}$ \\
\hline Estudios de género & 5 & $\begin{array}{l}\text { Líneas de investigación en educación que tienen como eje central } \\
\text { la categoría de género. }\end{array}$ \\
\hline Desarrollo cognitivo & 5 & $\begin{array}{l}\text { Investigaciones neurológicas y psicométricas sobre el desarrollo } \\
\text { cognitivo del cerebro y de las habilidades. }\end{array}$ \\
\hline Investigación científica & 4 & Física, biología, ciencias naturales y educación médica. \\
\hline Motivación & 4 & $\begin{array}{l}\text { Factores por los cuales los estudiantes y profesores se motivan y } \\
\text { reflejan ello en su forma de aprender o enseñar, al igual que los } \\
\text { factores que los desmotivan. }\end{array}$ \\
\hline Meta análisis & 4 & Conjunto de análisis con herramientas estadísticas. \\
\hline
\end{tabular}




\begin{tabular}{ccl}
\hline Categoría: Tema investigativo & Frecuencia & \multicolumn{1}{c}{ Descripción } \\
\hline Revisión de literatura & 3 & $\begin{array}{l}\text { Teorías sobre la enseñanza y literatura en educación, se estudian } \\
\text { diferentes autores y enfoques académicos. Revisiones históricas. }\end{array}$ \\
\hline Formación docente & 3 & $\begin{array}{l}\text { Experiencias desde los métodos para enseñar, los métodos y } \\
\text { enfoques pedagógicos para formar profesores y maestros. }\end{array}$ \\
\hline Líneas de investigación & 2 & $\begin{array}{l}\text { Se centran en los criterios de la investigación en educación, qué se } \\
\text { está investigando y en qué forma. }\end{array}$ \\
\hline Política educativa & 2 & Formas de financiación, política económica de la educación \\
\hline Educación infantil & 15 & $\begin{array}{l}\text { Investigaciones realizadas en las primeras edades, cursos principal- } \\
\text { mente del preescolar y la primaria. }\end{array}$ \\
\hline Educación secundaria y media & 2 & Adolescencia. \\
\hline Educación superior & 2 & $\begin{array}{l}\text { Estudiantes y profesores universitarios. Posgrados y estudios } \\
\text { posteriores. }\end{array}$ \\
\hline Educación especial & 1 & $\begin{array}{l}\text { Formación para estudiantes con capacidades cognitivas limitadas, } \\
\text { discapacidades, en condiciones cognitivas o físicas especiales. }\end{array}$ \\
\hline
\end{tabular}

Fuente: elaboración propia

Ahora, qué está investigando y publicando Colombia sobre educación:

Tabla 5. Tematización por artículos: Magis (UPJ), Papeles (UAN) y Pedagogía y Saberes (UPN)

\begin{tabular}{|c|c|c|}
\hline Categoría: Tema investigativo & Frecuencia & Descripción \\
\hline Líneas de investigación & 6 & $\begin{array}{l}\text { Se centran en los criterios de investigación en educación que se } \\
\text { están investigando y en qué forma. }\end{array}$ \\
\hline $\begin{array}{l}\text { Métodos y metodología de } \\
\text { enseñanza y aprendizaje }\end{array}$ & 6 & $\begin{array}{l}\text { Debates de las formas como se enseña y el manejo de diferentes } \\
\text { tipos de información. }\end{array}$ \\
\hline Sociedad y contextos & 6 & $\begin{array}{l}\text { Climas emocionales, la violencia y el conflicto, las relaciones de } \\
\text { origen y raza, las influencias familiares y las relaciones con los } \\
\text { padres. }\end{array}$ \\
\hline Revisión de literatura & 4 & $\begin{array}{l}\text { Teorías sobre la enseñanza y literatura en educación, se estudian } \\
\text { diferentes autores y enfoques académicos. Revisiones históricas. }\end{array}$ \\
\hline Desarrollo del aprendizaje & 3 & $\begin{array}{l}\text { Control del aprendizaje; experiencias y acompañamiento a los } \\
\text { estudiantes; educación formal e informal. }\end{array}$ \\
\hline Desempeño académico & 3 & $\begin{array}{l}\text { Formas y análisis sobre las evaluaciones y auto evaluaciones, } \\
\text { factores que afectan el rendimiento académico. }\end{array}$ \\
\hline Lenguaje y comunicación & 3 & $\begin{array}{l}\text { Análisis y artículos sobre la importancia del lenguaje, la comuni- } \\
\text { cación y sus medios; efectos de la lectura y la escritura. }\end{array}$ \\
\hline Tecnología y educación & 2 & $\begin{array}{l}\text { Las formas como diferentes plataformas tecnológicas, la Internet, } \\
\text { las redes sociales y otras tecnologías influyen en las maneras de } \\
\text { enseñar, aprender y desenvolverse en la escuela y el aula. }\end{array}$ \\
\hline Investigación científica & 1 & Física, biología, ciencias naturales y educación médica. \\
\hline Política educativa & 1 & Formas de financiación, política económica de la educación. \\
\hline Formación docente & 1 & $\begin{array}{l}\text { Experiencias desde los métodos para enseñar, los métodos y } \\
\text { enfoques pedagógicos para formar profesores y maestros. }\end{array}$ \\
\hline \multicolumn{3}{|c|}{ Niveles educativos señalados } \\
\hline Educación media & 2 & Adolescencia. \\
\hline Educación infantil & 1 & $\begin{array}{l}\text { Investigaciones realizadas en las primeras edades, cursos princi- } \\
\text { palmente del preescolar y la primaria. }\end{array}$ \\
\hline
\end{tabular}

Fuente: elaboración propia 
Con estas tematizaciones por artículos se pudo evidenciar que los temas en los cuales se enfocan las mejores revistas en educación, según Scimago, tratan en su gran mayoría sobre la categoría sociedad y contextos, que reúne las investigaciones sobre los diferentes ambientes emocionales, físicos y familiares en los cuales están inmersos tanto estudiantes como profesores, centrando el estudio en temas de violencia y conflictos, al mismo tiempo que trata temas sobre etnicidad, raza, niveles socioeconómicos y culturales diferentes. La mayoría de los artículos que pueden entrar en esta categoría son estudios de caso e investigaciones que observan las diferentes condiciones de vida. En segundo lugar, se encuentra la categoría de tecnología y educación, reafirmando lo que nuestra primera investigación arrojó sobre el alto impacto que está tomando esta área, tanto en la escritura como en la publicación, sobre todo frente a las comunidades y grupos investigativos que deben estarse formando alrededor de este reciente tema. Como tercera categoría, se encontró que los artículos se centran en buena parte sobre los métodos y metodología de enseñanza y aprendizaje, figurando, como hemos mencionado, que la categoría de ciencias sociales recoge precisamente esta revisión sobre la pedagogía.

Frente a esto, las revistas colombianas seleccionadas se encuentran un poco distantes de las primeras categorías halladas. Para el año 2014, la investigación y publicación sobre educación se centró en las líneas de investigación en educación, es decir, en observar qué

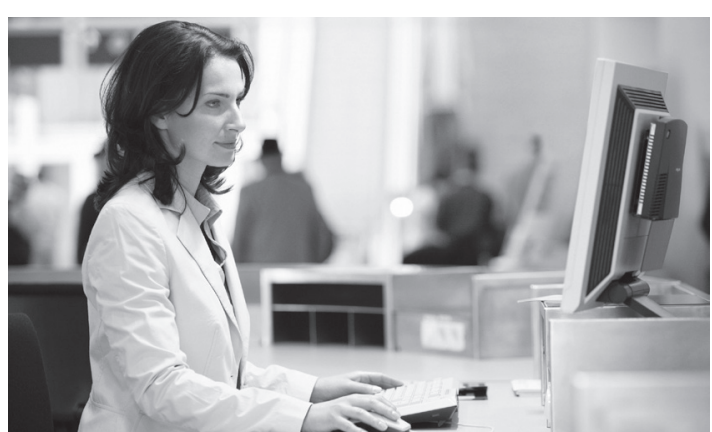

se está investigando y en qué forma; estas son, generalmente, revisiones epistemológicas sobre los criterios y principios investigativos, situación que se liga a los artículos encontrados que revisan la literatura sobre educación y las diferentes narrativas; por otro lado, en un acierto sobre los métodos y metodología de enseñanza y aprendizaje. Los investigadores colombianos que publicaron en esta selección, para el año mencionado, se enfocaron en cuestionar los métodos tradicionales que son aplicados en la escuela, sobre todo en materia de evaluaciones nacionales y extranjeras, desempeño académico y los roles que juegan los diferentes actores dentro de la escuela, tanto políticos como culturales.

\section{Directores e instituciones $^{4}$}

La mayoría de los directores de las revistas que están consideradas como las mejores en educación de Scimago son de nacionalidad estadounidense y tienen una formación académica extensa. Un factor común que encontramos en todos los directores es que tienen diversos estudios, entre ellos están: psicología, antropología, biología y ciencias exactas; no obstante, todos le han dado un énfasis especial a profundizar sus estudios en la educación. De esta manera, se evidencia que los directores no tienden a enfocar su investigación en un solo tema de estudio.

Los directores tienen varios artículos independientes y han recibido reconocimientos por algunos de estos. Dentro de su desarrollo profesional, además de ejercer su labor de directores, también se desempeñan como docentes universitarios. Un punto que es importante resaltar es que, al hacer una comparación de los estudios realizados por los directores de las revistas colombianas, se puede evidenciar que la mayoría de ellos ha buscado realizar sus estudios académicos en el exterior.

9 Este análisis se hace con el fin de comparar el proceso académico que tuvieron tanto los directores internacionales como los directores de las revistas colombianas seleccionadas. 


\section{Conclusiones}

Las primeras 15 revistas sobre educación escriben desde las disciplinas sociales, con enfoques multi e interdisciplinares. Además, la computación y la tecnología han tomado en poco tiempo un lugar relevante en temas educativos; esta afirmación realmente no es novedosa, sin embargo, suponer que en términos bibliométricos, un tema con tan poca 'trayectoria histórica' ya se encuentre posicionado entre las más importantes clasificaciones, significa, por un lado, que se ha escrito bastante sobre ello, y por otro, que se están conformando grandes comunidades académicas alrededor de este tema como una disciplina preeminente.

Por otra parte, se evidenció que el campo investigativo sobre educación en Colombia se encuentra poco comprometido a la hora de realizar indagaciones que puedan trascender en calidad. Se debe proponer innovar en los temas de consulta y aprovechar el potencial de sociedades y contextos.
En cuanto a las instituciones encargadas de publicar estas revistas, se puede concluir que la mayoría está vinculada a una universidad y que al hacer la publicación respectiva no son excluyentes con otras revistas. Dichas instituciones podrían funcionar como una base de datos que les ofrece a los profesionales y a los interesados en cada área de estudio obtener información de manera más eficiente.

Consideramos que, dadas las incongruencias y el peso del idioma de escritura de los artículos, las revistas colombianas estimadas en Scimago incurren en diferentes errores que no proporcionan una información verídica y decisiva para saber en qué nivel investigativo se encuentran frente a los temas dominantes de las revistas científicas más reconocidas en el área educativa. Por ello, este trabajo permite a los investigadores conocer no solamente las temáticas tratadas en las diferentes publicaciones clasificadas por Scimago, sino además los criterios más importantes que influyen en esa clasificación.

\section{Referencias}

AERA. (s.f). Aera 100. Recuperado el 8 de mayo de 2016, de AERA 100: http://www.aera.net/ AboutAERA/tabid/10062/Default.aspx

Albizu. (s.f). Albizu University. Recuperado el 8 de mayo de 2016, de ALBIZU UNIVERSITY: http://www.albizu.edu/Discover-CAU/Staff -and-Faculty-Directory/Staff-Directory/id/270

American Educational Research Association. (2014). Review of educational research, vol84.(3 y 4), pp. 324-639.Recuperado el 6 de abril de 2016. de: http://rer.sagepub.com/

American Psichologycal Association. (s.f). American Psichologycal Association. Recuperado el 9 de mayo de 2016, de American Psichologycal Association.

American Psychological Association Inc. (2014). Journal of Educational Psychology. Vol.106, (4), pp.901-1202. Recuperado el 6 de abril de 2016. de: http://www.apa.org/pubs/journals/edu/index. aspx

Arizona State University. (s.f). ASU. Recuperado el 9 de mayo de 2016, de ASU: https://education. asu.edu/faculty/steve-graham

Berkerly. (s.f). Berkerly Graduate school of education. Recuperado el 8 de Mayo de 2016, de Berkerly Graduate school of education: https:// gse.berkeley.edu/people/frank-c-worrell

Cerquera, L. B. (s.f). Linkedin. Recuperado el 9 de mayo de 2016, de Linkedin: https:// co.linkedin.com/in/luc\%C3\%ADa-bernalcerquera-ab710330

CIUP. (s.f). Universidad Pedagógica Nacional. Recuperado el 9 de mayo de 2016, de Universidad Pedagógica Nacional: http://investigaciones. pedagogica.edu.co 
Contreras, J. L. (s.f). Linkedin. Recuperado el 9 de mayo de 2016, de Linkedin: https:// co.linkedin.com/in/jose-leonardo-rincon -contreras-76882058

Elsevier Limited, Science direct. (2014). Computers and Education. Vol. 49, pp. 1-176. Recuperado el 13 de mayo de 2016, de: http:// www.elsevier.com/wps/find/journaldescription. cws_home/347/description\#description

Elsevier, Sciencie Direct. (2014). Handbook of the Economics of Education. Vol 4. Recuperado el 6 de abril de 2016. de: http://www.sciencedirect.com/science/handbooks/15740692/4g

Elsevier, Sciencie Direct. (2014). Learning and instruction.Vol.33, pp. 1-193. Recuperado el 6 de abril de 2016. de: http://www.elsevier.com/ wps/find/journaldescription.cws_home/956/ description\#description

Elsevier, Sciencie Direct. (2014). Learning and instruction.Vol.34, pp. 1-96. Recuperado el 6 de abril de 2016. de: http://www.elsevier.com/ wps/find/journaldescription.cws_home/956/ description\#description

Elsevier (s.f.) Scopus. Recuperado el 11 de Julio de 2016 de: http://www.americalatina.elsevier. com/corporate/es/scopus.php

Escritores colombianos. (s.f). Enrique Ferrer Corredor. Recuperado el 11 de Julio de 2016 de: http://escritorescolombianos.blogspot.com. co/2006/11/enrique-ferrer-corredor.html

Florida State University. (s.f). Florida State university college of education. Recuperado el 9 de mayo de 2016, de Florida State University College of Education: http://education.fsu.edu/ faculty-and-staff/dr-carolyn-herrington

Francis and Taylor. (s.f). Taylor and Francis Group. Recuperado el 8 de mayo de 2016, de Taylor and Francis Group: http://taylorandfrancis.com/about/

IE. (s.f). IE Business School. Recuperado el 9 de mayo de 2016, de IE Business School: http:// www.ie.edu/business-school/faculty-research/ faculty/enrique-ogliastri/

International Literacy Association. (2014). Reading Research Quarterly. Vol.49, (4), pp.365-487.
Recuperado el 13 de mayo de 2016. de: http:// www.reading.org/General/Publications/Journals/RRQ.aspx

McCarty, T. L. (s.f). WEBAPP. Recuperado el 9 de mayo de 2016, de WEBAPP: https://webapp4. asu.edu/directory/cv?id=716867

NARST (National association of research in science teaching). (2014). Journal of research in science teaching vol. 51 (7,8,9 y 10), pp.8211325. Recuperado el 6 de abril de 2016. de: http://onlinelibrary.wiley.com/journal/10.1002/ (ISSN)1098-2736

NARST. (s.f). NARST. Recuperado el 8 de mayo de 2016, de NARST: https://www.narst.org/ about/mission.cfm

Pontificia Universidad Javeriana. (s.f). Pontificia Universidad Javeriana. Recuperado el 9 de mayo de 2016, de Pontificia Universidad Javeriana: http://www.javeriana.edu.co/admisiones?gclid= CMLQ4t6R1cwCFYpZhgodIJ0HzQ

Pontificia Universidad Javeriana. (2014). Magis. Vol.7. (14), pp.15-176.Recuperado el 13 de mayo de 2016. de: http://revistas.javeriana.edu.co/ index.php/MAGIS

Routledge. (2014). Journal of the learning Science. Vol. 23, (3 y 4), pp.269-680.Recuperado el 13 de mayo de 2016. de: http://www.informaworld. $\mathrm{com} / \mathrm{smpp} /$ title $\sim$ content $=\mathrm{t} 775653672 \sim \mathrm{db}=$ all

Rudolph, J. L. (s.f). amscied. Recuperado el 8 de mayo de 2016, de amscied: http://www.amscied. net/About.html

SAGE Publications Ltd. (2014). Educational Researcher. Vol.43, (8,9), pp.381-464.Recuperado el 6 de abril de 2016. de: http://edr.sagepub. com/

SAGE Publications Ltda. (2014). American Educational Research Journal. Vol. 51, (6), pp.1056-1263.Recuperado el 6 de abril de 2016. de: http://aer.sagepub.com/

SAGE. (s.f). Sage Publishing. Recuperado el 8 de mayo de 2016, de SAGE PUBLISHING: https:// us.sagepub.com/en-us/sam/home

Science Direct. (s.f). Sciencie Direct. Recuperado el 9 de mayo de 2016, de Sciencie Direct: http:// www.sciencedirect.com 
Scimago Journal \& Country Rank, (2014). Journal Rankings, subject category: education, year: 2014. Recuperado el 3 de mayo de 2016. de: http://www.scimagojr.com/journalrank.php?are $\mathrm{a}=0$ \& category $=3304 \&$ country $=$ all $\&$ year $=2014 \&$ order $=$ sjr\&min $=0 \&$ min_type $=c d$

Scimago Journal \& Country Rank, (2014). Scimagoir. Recuperado el 9 de mayo de 2016, de Scimagoir: http://www.scimagoir.com/methodo logy.php

Springer Netherlands. (2014). User modelling and User adapted interaction.Vol.24, (3,4 y5), pp. 175-499. Recuperado el 13 de mayo de 2016. de: http://www.springer.com/computer/hci/jour nal/11257

Springer. (2014). International Journal of Computer-Supported Collaborative Learning. Vol.9, (3 y 4), pp. 237 - 475. Recuperado el 6 de abril de 2016, de: http://www.spri nger.com/education $+\% 26+$ language/learning $+\% 26+$ instruction/journal/11412

Springer. (s.f). Springer. Recuperado el 9 de mayo de 2016, de Springer: http://www.springer. com/la/about-springer

Stahl, G. (s.f). Gerry Stahl. Recuperado el 9 de mayo de 2016, de Gerry Stahl: http://gerrystahl. net

Taylor and Francis Ltda. (2014). Studies in Science Education. Vol. 50. (1,2), pp. 1-256. Recuperado el 6 de abril de 2016. de: http://www.tandfonline. com/toc/rsse20/current\#.V0ZKw5F97IU

Universidad Antonio Nariño. Revista de la Facultad de Educación Papeles. Recuperado el 11 de julio de 2016 de: http://revistas.uan.edu. co/index.php/papeles/issue/view/49

Universidad de los Andes. (s.f). Universidad de los Andes. Recuperado el 9 de mayo de 2016, de Universidad de los Andes: http://www.uniandes. edu.co

Universidad de los Andes. (2014). Academia: Revista Latinoamericana de Administración.
Vol. 26, (3). Recuperado el 13 de mayo de 2016. de:https://administracion.uniandes.edu.co/ index.php/es/component/k2/item/789-revistade-administraci\%C3\%B3n-academia-lograprimer-puestode-iberoam\%C3\%A9rica

Universidad de los Andes. (septiembre 3 de 2014). Revista de administración Academia logra primer puesto de Iberoamérica. Recuperado el 13 de mayo de: https://administracion.uniandes. edu.co/index.php/es/component/k2/item/789revista-de-administraci\% $\mathrm{C} 3 \% \mathrm{~B} 3 \mathrm{n}$-academialogra-primer-puestode-iberoam\%C3\%A9rica

Universidad Pedagógica de Colombia. (2014). Pedagogía y Saberes. Vol. 41, pp.1-120.Recuperado el 13 de mayo de: http://revistas.pedagogica. edu.co/index.php/PYS/index

University of Cambridge. (s.f). The Faculty of education. Recuperado el 9 de mayo de 2016, de: The Faculty of education: https://www.educ. cam.ac.uk/people/staff/vermunt/

University of Illinois. (s.f). Education at Illinois. Recuperado el 8 de mayo de 2016, de Education at Illinois: http://education.illinois.edu/faculty/ fouad

University of Leeds. (s.f). Faculty of Education, Social Sciences and Law. Recuperado el 8 de mayo de 2016, de Faculty of Education, Social Sciences and Law: http://www.education.leeds. ac.uk/people/academic/ryder

Willey. (s.f). Willey. Recuperado el 8 de mayo de 2016, de Willey: http://www.wiley.com/ WileyCDA/Brand/id-35.html

Willey-Blackwell. (2014). Child Development. Vol. 85. (4, 5 y 6), pp. 1323-2451. Recuperado el 6 de abril de 2016. de: http://onlinelibrary.wiley. com/journal/10.1111/(ISSN)1467-8624

Willey-Blackwell. (2014). Science Education. Vol.98. (4,5 y 6), pp. 549-1113. Recuperado el 6 de abril de 2016. de: http://onlinelibrary.wiley. com/journal/10.1002/(ISSN)1098-237X. 